\title{
Structural results for semigroup subsets defined by factorization properties dependent on $\Omega$ functions
}

\author{
Maciej Radziejewski
}

Received: 5 November 2013 / Accepted: 7 February 2014 / Published online: 21 March 2014

(C) The Author(s) 2014. This article is published with open access at Springerlink.com

\begin{abstract}
We study combinatorial properties of a class of subsets of semigroups with divisor theory motivated by the study of oscillations of counting functions of sets of algebraic integers with prescribed factorization properties.
\end{abstract}

Keywords Factorization lengths - Subsets defined by factorization properties · Semigroups with divisor theory $\cdot$ Krull monoids

\section{Introduction}

Semigroup subsets defined by factorization-related properties have been a subject of both structural (combinatorial) and quantitative (analytical) investigations. When the semigroup has an appropriate analytic structure (a positive-integer-valued norm with suitable properties) the counting function $A(x)$ of its subset $A$ may be defined as the number of non-associated elements of $A$ with norm not exceeding $x$. Quantitative theory of factorizations deals with the study of the counting functions of such subsets: the size of the main term, the size of the error term, and oscillatory behaviour of the error term. The first class of problems was essentially solved in the works of Fogels [2], Narkiewicz [10-12, 14, 15, etc.], Śliwa [23, etc.], and Geroldinger and Halter-Koch (e.g., [4, Chap. 9]). Kaczorowski [7] obtained refined asymptotics with an estimate of the size of the error term for a number of semigroup subsets, including the set $\boldsymbol{G}_{k}$ of elements with at most $k$ distinct factorization lengths in the ring of integers in

Communicated by László Márki.

M. Radziejewski $(\varangle)$

Faculty of Mathematics and Computer Science, Adam Mickiewicz University,

ul.Umultowska 87, 61-614 Poznań, Poland

e-mail: maciejr@amu.edu.pl 
an algebraic number field. General treatment of the problem, in an abstract setting, is again due to Geroldinger and Halter-Koch [4, Chap. 9]. Kaczorowski and Pintz [9] and Kaczorowski and Perelli [8] studied the oscillatory behaviour of the error terms for a number of subsets in rings of algebraic integers. Their methods are directly applicable to what we call " $\Omega$ sets of rank 0 " in the sequel. Kaczorowski and Pintz [9] noted that it is possible to extend their results to $\boldsymbol{G}_{k}$. This was done by the author [16-18] and by Schmid and the author [21] except for the case of $\boldsymbol{G}_{1}$, which was only treated conditionally, by assuming some conjectures of analytical or combinatorial character. This paper is a part of a series of papers where the present author settles the case of the set $\boldsymbol{G}_{1}$ in algebraic integers and, more generally, in a generalized Hilbert semigroup, as defined by Halter-Koch [5, Beispiel 4].

Let $H$ be a semigroup with divisor theory (cf. [3,5] and [4, Definition 2.4.1]) and a finite class group $G$. For $k \in \mathbf{N}$ let $\boldsymbol{G}_{k}$ denote the set of elements of $H$ with at most $k$ distinct factorization lengths in $H$. In this paper we show structural properties of $\boldsymbol{G}_{k}$ and of a class of other semigroup subsets that allow us to show, in the sequel to this paper, using analytic results from $[16,20]$, a theorem that implies the following (we omit the definitions of the "error term" and "oscillations" here):

Theorem If $H$ is a generalized Hilbert semigroup with more than 2 divisor classes, then for every $k \in \mathbf{N}$ the error term of the counting function of the set $\boldsymbol{G}_{k}$ has oscillations of logarithmic frequency and size $\sqrt{x}(\log x)^{-M}$ for some $M>0$.

We restrict this study to subsets $A$ of $H$ (or of the corresponding divisor semigroup) such that the value of the characteristic function of $A$ on an element $a$ depends only on the values of the functions $\Omega_{g}$ (the number of prime divisors in a given class) on $a$. We call such subsets " $\Omega$ sets". They correspond directly to "block-dependent factorization properties" considered by Geroldinger and Halter-Koch [4, Sect. 9.4]. A sufficiently regular $\Omega$ set $A$ may be expressed as a combination of simpler components, corresponding to what we call "cubes" in the sequel. When $H$ is equipped with a suitable analytic structure, the complex zeta function associated to a cube is essentially a combination of products of complex powers of various $L$-functions multiplied by polynomials in the logarithms of these $L$-functions, with coefficients being complex functions regular in a larger region. This allows for the determination of the size of the main term of the counting function corresponding to the cube, and to the result that the size main term of $A(x)$ is of the order

$$
x(\log x)^{\eta}(\log \log x)^{\delta},
$$

where the constants $\eta$ and $\delta$ depend on the combinatorial structure of $A$, specifically on what we call the $\operatorname{rank}(\operatorname{rk} A)$ and $\operatorname{degree}(\operatorname{deg} A)$ of $A$. This method was initiated by Narkiewicz [13, Theorem 9.4], cf. also Geroldinger and Halter-Koch [4, Theorem 9.4.3]. The values of the rank and degree of subsets defined by specific arithmetical properties are related to a number of interesting open problems in finite abelian groups, specifically in Zero-sum theory. For example, in the case when each divisor class contains a prime divisor, the degree of the set of irreducibles in $H$ is the Davenport constant of the class group $G$ [4, Chap. 6], rk $\boldsymbol{G}_{1}$ equals the constant denoted as $\mu(G)$, 
cf., e.g., [4, Sect. 6.7], and the degrees $\operatorname{deg} \boldsymbol{G}_{k}$ were considered by Śliwa [24] (in an equivalent form) and Radziejewski and Schmid [21].

Building upon the methods of Kaczorowski and Pintz [9] and Kaczorowski and Perelli [8] one can also show the existence of oscillations of the error term of $A(x)$ by showing that the associated zeta function is not regular in the half-plane $\Re s \geq \frac{1}{2}$ apart from the real line. The problem is that in most cases neither the combinatorial structure of $A$ (the expression of $A$ as a combination of cubes), nor the analytic shape of the zeta functions of cubes, nor the multiplicities of zeros of the related $L$-functions are known completely. Roughly speaking, the rank of a cube affects the exponents of the complex powers of $L$-functions in the associated zeta function, and its degree is the degree of the polynomial in the logarithms. Our approach is to concentrate on the principal summands of zeta functions of cubes of some rank $r$ and degree $d>0$ (so that logarithms are necessarily involved) and show that similar terms do not cancel out. This motivates the notion of $(r, d)$-singular sets introduced in Sect. 3.4—with suitable analytical structure we are able to show the existence of oscillations precisely for such sets. The main result of the paper is the complete characterization of $(r, d)$-singular $\Omega$ sets closed upon in-class divisors (Theorem 11), that allows us to treat $\boldsymbol{G}_{1}$. Essentially, previous methods allowed us to show the existence of oscillations when $\operatorname{deg} A>0$, in which case $A$ is (rk $A, \operatorname{deg} A$ )-singular (Fact 7).

Sections 3.1 through 3.3 provide the necessary basis for the rest of the paper. A number of results and methods there are known, but we include some of the results for completeness and some, because they were not stated explicitly in the form that we need further. It seems that the rank and degree did not have a general name previously, and that they were not considered for sets other than those expressible as finite combinations of cubes. The implication $(i) \Rightarrow(i i)$ in Proposition 6 is due to Geroldinger and Halter-Koch [4, Proposition 9.4.2.3] who considered a condition similar to $l(A)=1$, cf. [4, Definition 9.4.1.3]. The implication (ii) $\Rightarrow$ (iii) in Proposition 6 is essentially due to Kaczorowski [7, Sect. 8]. Lemma 2.3 was shown by Geroldinger and HalterKoch [4, Proposition 9.4.2.4], but we still provide a proof, because of slightly different hypotheses.

\section{Notation}

The order of a group element $g$ is denoted as ord $g$. For sets $A$ and $B$ we denote by $A^{B}$ is the set of functions from a set $B$ to a set $A$, and by $|A|$ the cardinality of $A$. For a set $X$ we let $\mathcal{F}(X)$ denote the free (multiplicative) abelian monoid generated by a set $X$. For $S \in \mathcal{F}(X)$ we put:

$$
S=\prod_{x \in X} x^{v_{x}(S)}, \quad \operatorname{Supp}(S)=\left\{x \in X: \mathrm{v}_{x}(S)>0\right\} .
$$

Let $G$ be a finite abelian group (written additively), $G_{0} \subseteq G$. For $S \in \mathcal{F}\left(G_{0}\right)$ we put, following [4],

$$
|S|=\sum_{g \in G_{0}} \mathrm{v}_{g}(S) \in \mathbf{N}_{0}, \quad \sigma(S)=\sum_{g \in G_{0}} \mathrm{v}_{g}(S) g \in G,
$$


and, for $U \subseteq G_{0}, S \in \mathcal{F}\left(G_{0} \backslash U\right) \subseteq \mathcal{F}\left(G_{0}\right), y \in G$ and $d \in \mathbf{N}_{0}$,

$$
\begin{aligned}
\Omega_{y}(U, S) & =\left\{S^{\prime} \in S \mathcal{F}(U): \sigma\left(S^{\prime}\right)=y\right\} \\
\Omega_{y}(U, S, d) & =\left\{S^{\prime} \in \Omega_{y}(U, S): \mathrm{v}_{g}\left(S^{\prime}\right) \geq d \text { for all } g \in U\right\} .
\end{aligned}
$$

In particular we have

$$
\mathcal{F}\left(G_{0}\right)=\bigcup_{y \in G} \Omega_{y}\left(G_{0}, 1\right) .
$$

We let $\mathrm{S}\left(G_{0}\right)$ denote the set of all triples $(U, S, y)$ such that $U \subseteq G_{0}, S \in \mathcal{F}\left(G_{0} \backslash U\right) \subseteq$ $\mathcal{F}\left(G_{0}\right), y \in G$, and $\Omega_{y}(U, S) \neq \emptyset$. We refer to such non-empty sets $\Omega_{y}(U, S)$ as cubes (over $G_{0}$ ), and a cube contained in another cube a sub-cube of the latter. For a set $U \subseteq G_{0}$ the cube $\Omega_{0}(U, 1)$ is also denoted as $\mathcal{B}(U)$. It is a submonoid of $\mathcal{F}(U)$ and it is called the block monoid over $U$. For any subset $\mathcal{A} \subseteq \mathcal{F}\left(G_{0}\right)$ we define its in-class divisor closure as

$$
\operatorname{Div}(\mathcal{A})=\left\{S \in \mathcal{F}\left(G_{0}\right): S \mid S^{\prime} \text { for some } S \in \mathcal{A}, \sigma(S)=\sigma\left(S^{\prime}\right)\right\}
$$

For a set $X$ we consider the set $\overline{\mathcal{F}(X)}=\left(\mathbf{N}_{0} \cup\{+\infty\}\right)^{X}$ with the product order $\leq$. We treat $\mathcal{F}(X)$ as a subset of $\overline{\mathcal{F}(X)}$. For elements of $\mathcal{F}(X)$ the order $\leq$ coincides with divisibility, but we still use the symbol $\leq$ wherever the elements of $\overline{\mathcal{F}(X)}$ might be involved. However, for compatibility with the notation employed for $\mathcal{F}(X)$, we denote the initial element of $\overline{\mathcal{F}(X)}$ as 1 and, for $a \in \overline{\mathcal{F}(X)}$, we write $\mathrm{v}_{x}(a)$ instead of $a(x)$. For a sequence $\left(b_{n}\right)$ in $\overline{\mathcal{F}(X)}$ we denote by $\sup _{n}\left(b_{n}\right)$ the element $b^{\prime} \in \overline{\mathcal{F}(X)}$ defined by $\mathrm{v}_{x}\left(b^{\prime}\right)=\sup _{n}\left(\mathrm{v}_{x}\left(b_{n}\right)\right), x \in X$.

We recall that a subset of a partially ordered set is called an antichain if no two of its elements are comparable. If $a, b$ belong to a partially ordered set $A$, then we write

$$
[a, b]=\{x \in A: a \leq x \leq b\}
$$

For partially ordered sets $A_{1}$ and $A_{2}$ we define the lexicographic order in $A_{1} \times A_{2}$ as

$$
\left(a_{1}, a_{2}\right) \leq\left(b_{1}, b_{2}\right) \Leftrightarrow a_{1}<b_{1} \vee\left(a_{1}=b_{1} \wedge a_{2} \leq b_{2}\right) .
$$

The product order in a product of partially ordered sets $\prod_{i \in I} A_{i}$ is of course

$$
\left(a_{i}\right) \leq\left(b_{i}\right) \Leftrightarrow \bigwedge_{i \in I} a_{i} \leq b_{i} .
$$

\section{Sets of sequences over a finite abelian group}

3.1 Basic properties of cubes

Lemma 1 Let $\left(U_{1}, S_{1}, y\right),\left(U_{2}, S_{2}, y_{2}\right) \in \mathrm{S}\left(G_{0}\right)$. 
1. If

$$
y=y_{2} \quad \text { and } \quad \mathbf{v}_{g}\left(S_{1}\right)=\mathrm{v}_{g}\left(S_{2}\right) \text { for all } g \in G_{0} \backslash\left(U_{1} \cup U_{2}\right),
$$

then we have

$$
\Omega_{y}\left(U_{1}, S_{1}\right) \cap \Omega_{y_{2}}\left(U_{2}, S_{2}\right)=\Omega_{y}\left(U_{1} \cap U_{2}, \operatorname{lcm}\left(S_{1}, S_{2}\right)\right) .
$$

2. If $\Omega_{y}\left(U_{1}, S_{1}\right) \cap \Omega_{y_{2}}\left(U_{2}, S_{2}\right) \neq \emptyset$, then (2) holds.

Proof For $S \in \mathcal{F}\left(G_{0}\right)$ we have $S \in \Omega_{y}\left(U_{1}, S_{1}\right) \cap \Omega_{y_{2}}\left(U_{2}, S_{2}\right)$ if and only if $\sigma(S)=$ $y=y_{2}$ and

$$
\mathrm{v}_{g}(S)= \begin{cases}\mathrm{v}_{g}\left(S_{1}\right), & g \in G_{0} \backslash U_{1}, \\ \mathrm{v}_{g}\left(S_{2}\right), & g \in G_{0} \backslash U_{2} .\end{cases}
$$

This can be rewritten as the conjunction of (2) and

$$
\begin{aligned}
\sigma(S) & =y, \\
\mathrm{v}_{g}(S) & =\mathrm{v}_{g}\left(\operatorname{lcm}\left(S_{1}, S_{2}\right)\right) \quad \text { for all } g \in G_{0} \backslash\left(U_{1} \cap U_{2}\right) .
\end{aligned}
$$

The existence of such $S$ implies (2). Moreover, as (4) is equivalent to $S \in \Omega_{y}\left(U_{1} \cap\right.$ $\left.U_{2}, \operatorname{lcm}\left(S_{1}, S_{2}\right)\right)$, the condition (2) implies the equality (3).

Lemma 2 1. If $(U, S, y) \in \mathrm{S}\left(G_{0}\right)$, then for every $d \in \mathbf{N}_{0}$ we have

$$
\Omega_{y}(U, S, d) \neq \emptyset .
$$

Moreover, for every $S_{0} \in \mathcal{F}\left(G_{0}\right)$ such that $S_{0} \mid S$, and every $S^{\prime} \in \Omega_{y}\left(U, S_{0}\right)$, $d \geq \max _{g \in G_{0} \backslash U} \mathrm{v}_{g}\left(S^{\prime}\right), S^{\prime \prime} \in \Omega_{y}(U, S, d)$ we have $S^{\prime} \mid S^{\prime \prime}$.

2. For $\left(U_{1}, S_{1}, y_{1}\right),\left(U_{2}, S_{2}, y_{2}\right) \in \mathrm{S}\left(G_{0}\right)$ we have $\Omega_{y_{1}}\left(U_{1}, S_{1}\right) \subseteq \Omega_{y_{2}}\left(U_{2}, S_{2}\right)$ if and only if $y_{1}=y_{2}, U_{1} \subseteq U_{2}$ and $\mathrm{v}_{g}\left(S_{1}\right)=\mathrm{v}_{g}\left(S_{2}\right)$ for all $g \in G_{0} \backslash U_{2}$.

3. If $(U, S, y) \in \mathrm{S}\left(G_{0}\right), d \in \mathbf{N}_{0},\left(U_{1}, S_{1}, y_{1}\right), \ldots,\left(U_{n}, S_{n}, y_{n}\right) \in \mathrm{S}\left(G_{0}\right)$ and

$$
\Omega_{y}(U, S, d) \subseteq \bigcup_{i=1}^{n} \Omega_{y_{i}}\left(U_{i}, S_{i}\right),
$$

then $\Omega_{y}(U, S) \subseteq \Omega_{y_{i}}\left(U_{i}, S_{i}\right)$ for some $i \in\{1, \ldots, n\}$.

4. If $\left(U_{1}, S_{1}, y_{1}\right), \ldots,\left(U_{n}, S_{n}, y_{n}\right) \in \mathbf{S}\left(G_{0}\right)$ are pairwise distinct, then the characteristic functions of the cubes $\Omega_{y_{i}}\left(U_{i}, S_{i}\right)$ are linearly independent.

Proof 1. Let $S^{\prime} \in \Omega_{y}(U, S)$. Then for every $d$ we have

$$
S^{\prime} \prod_{g \in U} g^{d \text { ord } g} \in \Omega_{y}(U, S, d),
$$

and the "moreover" part is by definition. 
2. To see $\Rightarrow$ we take $d>\max _{g \in G_{0}} \mathrm{v}_{g}\left(S_{2}\right)$ in 1 . and obtain $A \in \Omega_{y_{1}}\left(U_{1}, S_{1}\right)$ with $\mathrm{v}_{g}(A) \neq \mathrm{v}_{g}\left(S_{2}\right), g \in U_{1}$. If $\Omega_{y_{1}}\left(U_{1}, S_{1}\right) \subseteq \Omega_{y_{2}}\left(U_{2}, S_{2}\right)$, then $A \in \Omega_{y_{2}}\left(U_{2}, S_{2}\right)$ implies $\mathrm{v}_{g}(A)=\mathrm{v}_{g}\left(S_{2}\right), g \in G_{0} \backslash U_{2}$, so $U_{1} \subseteq U_{2}$ and the rest follows from Lemma 1.2. The implication $\Leftarrow$ follows from Lemma 1.1 .

3. Suppose, as we may, that $d>\max _{g \in G_{0}} \max _{i} \mathrm{v}_{g}\left(S_{i}\right)$. An element $A \in \Omega_{y}(U, S, d)$ exists by 1 . We have $A \in \Omega_{y_{i}}\left(U_{i}, S_{i}\right)$ for some $i \in\{1, \ldots, n\}$, hence $U \subseteq U_{i}$, and so $\mathrm{v}_{g}(S)=\mathrm{v}_{g}(A)=\mathrm{v}_{g}\left(S_{i}\right)$ for all $g \in G_{0} \backslash U_{i}$, as well as $y=\sigma(A)=y_{i}$, hence $\Omega_{y}(U, S) \subseteq \Omega_{y_{i}}\left(U_{i}, S_{i}\right)$ by 2 .

4. Suppose, as we may, that $U_{1}$ is not a proper subset of any of the $U_{i}, i=2, \ldots, n$. If we had $\Omega_{y_{1}}\left(U_{1}, S_{1}\right) \subseteq \Omega_{y_{i}}\left(U_{i}, S_{i}\right)$ for any $i \in\{2, \ldots, n\}$, then by 2 . we would have $y_{1}=y_{i}, U_{1} \subseteq U_{i}$, and $\mathrm{v}_{g}\left(S_{1}\right)=\mathrm{v}_{g}\left(S_{i}\right)$ for all $g \in G_{0} \backslash U_{i}$ for some $i \geq 2$. Hence $U_{i}=U_{1}$ by maximality of $U_{1}$, and so $S_{i}=S_{1}$, contrary to the assumption of the triples being distinct. Hence we have $\Omega_{y_{1}}\left(U_{1}, S_{1}\right) \nsubseteq$ $\Omega_{y_{i}}\left(U_{i}, S_{i}\right), i=2, \ldots, n$, and so $\Omega_{y_{1}}\left(U_{1}, S_{1}\right) \nsubseteq \bigcup_{i=2}^{n} \Omega_{y_{i}}\left(U_{i}, S_{i}\right)$ by 3 . The characteristic function of $\Omega_{y_{1}}\left(U_{1}, S_{1}\right)$ is thus linearly independent of the others and the assertion follows by induction.

\subsection{Rank and degree}

If $\mathcal{A}$ is a non-empty subset of $\mathcal{F}\left(G_{0}\right)$, we define its rank rk $\mathcal{A}$ as the smallest $r \in$ $\left\{0, \ldots,\left|G_{0}\right|\right\}$ such that there exist $n \in \mathbf{N}$ and $\left(U_{1}, S_{1}, y_{1}\right), \ldots,\left(U_{n}, S_{n}, y_{n}\right) \in \mathrm{S}\left(G_{0}\right)$ satisfying

$$
\mathcal{A} \subseteq \bigcup_{i=1}^{n} \Omega_{y_{i}}\left(U_{i}, S_{i}\right)
$$

and $\max _{i}\left|U_{i}\right| \leq r$. Such an $r$ always exists by (1). We put $\operatorname{rk} \emptyset=-\infty$.

Lemma 3 1. For $\mathcal{A}, \mathcal{B} \in \mathcal{F}\left(G_{0}\right)$ we have $\operatorname{rk}(\mathcal{A} \cup \mathcal{B})=\max (\operatorname{rk} \mathcal{A}$, rk $\mathcal{B})$.

2. If $\mathcal{A} \subseteq \mathcal{B}$, then $\mathrm{rk} \mathcal{A} \leq \mathrm{rk} \mathcal{B}$.

3. If $(U, S, y) \in \mathrm{S}\left(G_{0}\right)$, then we have $\mathrm{rk} \Omega_{y}(U, S)=|U|$.

4. If $\mathcal{A} \subseteq \mathcal{F}\left(G_{0}\right)$ is contained in a finite union $\bigcup_{i=1}^{n} \Omega_{y_{i}}\left(U_{i}, S_{i}\right)$, where $n \in \mathbf{N}$ and $\left(U_{1}, S_{1}, y_{1}\right), \ldots,\left(U_{n}, S_{n}, y_{n}\right) \in \mathrm{S}\left(G_{0}\right)$, then

$$
\operatorname{rk} \mathcal{A}=\max _{1 \leq i \leq n} \operatorname{rk}\left(\mathcal{A} \cap \Omega_{y_{i}}\left(U_{i}, S_{i}\right)\right)
$$

Proof 1. and 2. follow from the definition of rank.

3. Let $r=|U|$. The inequality $\mathrm{rk} \Omega_{y}(U, S) \leq r$ is trivial by the definition of rank.

The converse inequality follows from the definition and Lemma 2.3.

4. Follows from 1. and

$$
\mathcal{A}=\bigcup_{i=1}^{n}\left(\mathcal{A} \cap \Omega_{y_{i}}\left(U_{i}, S_{i}\right)\right) .
$$


Next we define the degree $\operatorname{deg} \mathcal{A}$ of a set $\mathcal{A} \subseteq \mathcal{F}\left(G_{0}\right)$ as the supremum of all values of $|S|$ over the elements $(U, S, y)$ of the set

$$
\left\{(U, S, y) \in \mathrm{S}\left(G_{0}\right): \operatorname{rk}\left(\mathcal{A} \cap \Omega_{y}(U, S)\right)=\operatorname{rk} \mathcal{A}\right\}
$$

We note that $\Omega_{y}\left(G_{0}, 1\right)$ satisfies the condition in (5) for some $y \in G$ by (1) and Lemma 3.4, so $\operatorname{deg} \mathcal{A} \geq 0$. In particular we have $\operatorname{deg} \emptyset=+\infty$.

Lemma 4 1. For $\mathcal{A}, \mathcal{B} \subseteq \mathcal{F}\left(G_{0}\right)$ we have

$$
\operatorname{deg}(\mathcal{A} \cup \mathcal{B})= \begin{cases}\max (\operatorname{deg} \mathcal{A}, \operatorname{deg} \mathcal{B}), & \text { if } \mathrm{rk} \mathcal{A}=\operatorname{rk} \mathcal{B}, \\ \operatorname{deg} \mathcal{B}, & \text { if } \mathrm{rk} \mathcal{A}<\mathrm{rk} \mathcal{B}\end{cases}
$$

2. For $\mathcal{A} \subseteq \mathcal{B} \subseteq \mathcal{F}\left(G_{0}\right)$ we have either $\mathrm{rk} \mathcal{A}<\mathrm{rk} \mathcal{B}$, or $\mathrm{rk} \mathcal{A}=\operatorname{rk} \mathcal{B}$ and $\operatorname{deg} \mathcal{A} \leq \operatorname{deg} \mathcal{B}$, i.e. the mapping $\mathcal{A} \mapsto(\operatorname{rk} \mathcal{A}, \operatorname{deg} \mathcal{A})$ is monotone with respect to the partial order defined by inclusion of subsets of $\mathcal{F}\left(G_{0}\right)$, and lexicographic order for (rk, deg) pairs.

3. If $(U, S, y) \in \mathrm{S}\left(G_{0}\right)$, then $\operatorname{deg} \Omega_{y}(U, S)=|S|$.

4. If $\mathcal{A} \subseteq \mathcal{F}\left(G_{0}\right)$ is contained in a finite union $\bigcup_{i=1}^{n} \Omega_{y_{i}}\left(U_{i}, S_{i}\right)$, where $n \in \mathbf{N}$ and $\left(U_{1}, S_{1}, y_{1}\right), \ldots,\left(U_{n}, S_{n}, y_{n}\right) \in \mathrm{S}\left(G_{0}\right)$ are such that

$$
\operatorname{rk} \mathcal{A}=\max _{1 \leq i \leq n}\left|U_{i}\right|
$$

then

$$
\operatorname{deg} \mathcal{A}=\max _{i \in J}\left|S_{i}\right|
$$

where $J=\left\{i \in\{1, \ldots, n\}: \operatorname{rk}\left(\mathcal{A} \cap \Omega_{y_{i}}\left(U_{i}, S_{i}\right)\right)=\operatorname{rk} \mathcal{A}\right\}$.

5. If $\mathcal{A} \subseteq \mathcal{F}\left(G_{0}\right)$ and $\mathcal{A} \neq \emptyset$, then $\operatorname{deg} \mathcal{A} \in \mathbf{N}_{0}$.

Proof 1. Let $\operatorname{Deg}(\mathcal{A})$ denote the set (5). For every $(U, S, y) \in \mathrm{S}\left(G_{0}\right)$ the equality

$$
\operatorname{rk}\left(\Omega_{y}(U, S) \cap(\mathcal{A} \cup \mathcal{B})\right)=\operatorname{rk}(\mathcal{A} \cup \mathcal{B})
$$

is equivalent, by Lemma 3.1, to

$$
\max \left(\mathrm{rk}\left(\Omega_{y}(U, S) \cap \mathcal{A}\right), \operatorname{rk}\left(\Omega_{y}(U, S) \cap \mathcal{B}\right)\right)=\max (\operatorname{rk} \mathcal{A}, \mathrm{rk} \mathcal{B})
$$

If $\operatorname{rk} \mathcal{A}=\operatorname{rk} \mathcal{B}$, then (6) reduces, by Lemma 3.2, to the alternative

$$
\operatorname{rk}\left(\Omega_{y}(U, S) \cap \mathcal{A}\right)=\operatorname{rk} \mathcal{A} \quad \text { or } \quad \operatorname{rk}\left(\Omega_{y}(U, S) \cap \mathcal{B}\right)=\operatorname{rk} \mathcal{B}
$$

so $\operatorname{Deg}(\mathcal{A} \cup \mathcal{B})=\operatorname{Deg}(\mathcal{A}) \cup \operatorname{Deg}(\mathcal{B})$. If $\operatorname{rk} \mathcal{A}<\operatorname{rk} \mathcal{B}$, then (6) similarly becomes

$$
\operatorname{rk}\left(\Omega_{y}(U, S) \cap \mathcal{B}\right)=\operatorname{rk} \mathcal{B}
$$


so $\operatorname{Deg}(\mathcal{A} \cup \mathcal{B})=\operatorname{Deg}(\mathcal{B})$. The assertion follows.

2. Follows from 1 .

3. Let $r=|U|$ and $d=|S|$. We have $\operatorname{deg} \Omega_{y}(U, S) \geq d$ by definition. To see that $\operatorname{deg} \Omega_{y}(U, S) \leq d$, we note that for any $\left(U^{\prime}, S^{\prime}, y^{\prime}\right) \in \mathrm{S}\left(G_{0}\right)$ such that

$$
\operatorname{rk}\left(\Omega_{y}(U, S) \cap \Omega_{y^{\prime}}\left(U^{\prime}, S^{\prime}\right)\right)=\operatorname{rk} \Omega_{y}(U, S)
$$

we must have $\Omega_{y}(U, S) \cap \Omega_{y^{\prime}}\left(U^{\prime}, S^{\prime}\right) \neq \emptyset$, so $y^{\prime}=y$ and $\left|U \cap U^{\prime}\right|=r$ by Lemmas 1 and 3.3, hence $U \subseteq U^{\prime}$ and, again by Lemma $1,\left|S^{\prime}\right| \leq d$.

4. By 1. we have

$$
\operatorname{deg} \mathcal{A}=\max _{i \in J} \operatorname{deg}\left(\mathcal{A} \cap \Omega_{y_{i}}\left(U_{i}, S_{i}\right)\right)
$$

Let $i \in J$ and $r=\operatorname{rk} \mathcal{A}=\operatorname{rk}\left(\mathcal{A} \cap \Omega_{y_{i}}\left(U_{i}, S_{i}\right)\right)$. Obviously

$$
\operatorname{rk}\left(\mathcal{A} \cap \Omega_{y_{i}}\left(U_{i}, S_{i}\right) \cap \Omega_{y_{i}}\left(U_{i}, S_{i}\right)\right)=r,
$$

so

$$
\operatorname{deg}\left(\mathcal{A} \cap \Omega_{y_{i}}\left(U_{i}, S_{i}\right)\right) \geq\left|S_{i}\right|
$$

by the definition of degree. We also have rk $\Omega_{y_{i}}\left(U_{i}, S_{i}\right) \leq r$ by assumption and Lemma 3.3, and rk $\Omega_{y_{i}}\left(U_{i}, S_{i}\right) \geq r$ by Lemma 3.2. Hence

$$
\operatorname{rk}\left(\mathcal{A} \cap \Omega_{y_{i}}\left(U_{i}, S_{i}\right)\right)=\operatorname{rk} \Omega_{y_{i}}\left(U_{i}, S_{i}\right)
$$

so

$$
\operatorname{deg}\left(\mathcal{A} \cap \Omega_{y_{i}}\left(U_{i}, S_{i}\right)\right) \leq \operatorname{deg} \Omega_{y_{i}}\left(U_{i}, S_{i}\right)=\left|S_{i}\right|
$$

5. Follows from 4., because a finite union $\bigcup_{i=1}^{n} \Omega_{y_{i}}\left(U_{i}, S_{i}\right)$ satisfying the assumptions of 4 . must exist by the definition of rank.

\subsection{Sets with a finite number of layers}

For every $\mathcal{A} \subseteq \mathcal{F}\left(G_{0}\right)$ we define $l(\mathcal{A})$, the number of layers of $\mathcal{A}$, as the maximum length $l$ of an interleaved chain of divisors $a_{1}\left|b_{1}\right| a_{2}\left|b_{2}\right| \ldots\left|b_{l-1}\right| a_{l}$ in $\mathcal{F}\left(G_{0}\right)$ such that $a_{1}, a_{2}, \ldots, a_{l} \in \mathcal{A}, b_{1}, b_{2}, \ldots, b_{l-1} \notin \mathcal{A}$, and there exists some $y \in G$ such that $\sigma\left(a_{i}\right)=\sigma\left(b_{j}\right)=y$ for $i=1, \ldots, l, j=1, \ldots, l-1$. In particular we have $l(\emptyset)=0$. We need one more auxiliary result before giving a complete characterization of sets with a finite number of layers. 
Lemma 5 If $X$ is a finite set, then every antichain in $\overline{\mathcal{F}(X)}$ is finite.

Proof For an antichain $A \subseteq \overline{\mathcal{F}(X)}$ let $I(A)$ denote the set of such $x \in X$ that there exists an $a \in A$ that satisfies $\mathrm{v}_{x}(a)=+\infty$. Suppose there exists an infinite antichain $A \subseteq \overline{\mathcal{F}(X)}$ and that $|X|$ is the smallest possible number for which this happens. Suppose further that $|I(A)|$ is the smallest possible given this $X$. Let $a \in A$. We have

$$
A=\{a\} \cup \bigcup_{x \in X} A_{x},
$$

where $A_{x}=\left\{b \in A: \mathrm{v}_{x}(b)<\mathrm{v}_{x}(a)\right\}$. For $x \in I(A)$ we have $I\left(A_{x}\right) \subseteq I(A) \backslash\{x\}$, so $A_{x}$ is finite by assumption. For $x \in X \backslash I(A)$ we have

$$
A_{x}=\bigcup_{j=0}^{\mathrm{v}_{x}(a)-1} A_{x, j}
$$

where $A_{x, j}=\left\{b \in A: \mathrm{v}_{x}(b)=j\right\}$. For $x, j$ as above let $A_{x, j}^{\prime}$ denote the projection of $A_{x, j}$ onto $\overline{\mathcal{F}(X \backslash\{x\})}$. Then $A_{x, j}^{\prime} \subseteq \overline{\mathcal{F}(X \backslash\{x\})}$ is an antichain and $|X \backslash\{x\}|<|X|$, so $A_{x, j}^{\prime}$ is finite by assumption, and so is $A_{x, j}$, as equinumerous to $A_{x, j}^{\prime}$. Hence $A$ is finite, a contradiction.

Proposition 6 Let $\mathcal{A} \subseteq \mathcal{F}\left(G_{0}\right)$. The following conditions are equivalent:

(i) $l(\mathcal{A})<+\infty$

(ii) $\mathcal{A}=\bigcup_{i=1}^{m} B_{i}$ for some $m \in \mathbf{N}_{0}$ and sets $B_{i}$ of the form

$$
B_{i}=\left\{a \in \mathcal{F}\left(G_{0}\right) \cap\left[\alpha_{i, 1}, \alpha_{i, 2}\right]: \sigma(a)=y_{i}\right\},
$$

where $y_{i} \in G$, and $\alpha_{i, 1}, \alpha_{i, 2} \in \overline{\mathcal{F}\left(G_{0}\right)}, i=1, \ldots, m$.

(iii) The characteristic function of $\mathcal{A}$ can be represented in the form

$$
\mathcal{A}=\sum_{j=1}^{n} \gamma_{j} \cdot \Omega_{g_{j}}\left(U_{j}, S_{j}\right),
$$

where $n \in \mathbf{N}_{0}$, sets are tacitly identified with their characteristic functions, the triples $\left(U_{j}, S_{j}, g_{j}\right) \in \mathrm{S}\left(G_{0}\right)$ are pairwise distinct, and $\gamma_{j} \in \mathbf{Z} \backslash\{0\}, j=1, \ldots, n$.

The representation (7) is then unique up to order. If $\mathcal{A} \neq \emptyset$, then we have

$$
\operatorname{rk} \mathcal{A}=\max _{j}\left|U_{j}\right|, \quad \operatorname{deg} \mathcal{A}=\max _{j:\left|U_{j}\right|=\mathrm{rk} \mathcal{A}}\left|S_{j}\right|,
$$

and $\gamma_{j}=1$ for all $j \in\{1, \ldots, n\}$ such that the cube $\Omega_{g_{j}}\left(U_{j}, S_{j}\right)$ is inclusion-maximal among others.

Proof $(i) \Rightarrow($ ii $)$. First we reduce the general case to a specific one. The intersection of $\mathcal{A}$ with each subset $\Omega_{y}\left(G_{0}, 1\right)$ in (1) may be treated independently, so we can 
assume $\mathcal{A} \subseteq \Omega_{y}\left(G_{0}, 1\right)$ for some fixed $y \in G$. We set $Y=\Omega_{y}\left(G_{0}, 1\right)$. Let $\mathcal{A}_{1}$ denote the set of all $a_{1} \in \mathcal{A}$ such that for some $a_{2}, \ldots, a_{l} \in \mathcal{A}$ and $b_{1}, b_{2}, \ldots, b_{l-1} \in Y \backslash \mathcal{A}$ we have $a_{1}\left|b_{1}\right| a_{2}\left|b_{2}\right| \ldots\left|b_{l-1}\right| a_{l}$. We have $l\left(\mathcal{A}_{1}\right)=1$ and $l\left(\mathcal{A} \backslash \mathcal{A}_{1}\right)=l-1$, so it is enough to prove the assertion in the case $l(\mathcal{A})=1$ and use induction. Finally, given $l(\mathcal{A})=1$, we note that the set $\mathcal{A}^{\prime}=\operatorname{Div}(\mathcal{A}) \backslash \mathcal{A}$ satisfies $\operatorname{Div}\left(\mathcal{A}^{\prime}\right)=\mathcal{A}^{\prime}$, so $\mathcal{A}=\operatorname{Div}(\mathcal{A}) \backslash \operatorname{Div}\left(\mathcal{A}^{\prime}\right)$. As the family of sets expressible in the form required in $(\mathrm{ii})$ is closed upon set difference, it is enough to prove the assertion in the case when

$$
\mathcal{A}=\operatorname{Div}(\mathcal{A}) \subseteq Y
$$

Let $\mathcal{B}=\left\{b \in \overline{\mathcal{F}\left(G_{0}\right)}: Y \cap[1, b] \subseteq \mathcal{A}\right\}$ and let $\mathcal{B}_{\text {max }}$ denote the set of maximal elements of $\mathcal{B}$. In order to obtain

$$
\mathcal{A}=\bigcup_{b \in \mathcal{B}_{\max }} Y \cap[1, b]
$$

we need to show that for every $a \in \mathcal{A}$ there exists some $b^{\prime} \in \mathcal{B}_{\max }$ such that $a \leq b^{\prime}$, so we assume otherwise. We have $\mathcal{A} \subseteq \mathcal{B}$, so, starting with $b_{1}=a$, we can construct an increasing sequence in $\mathcal{B}$ :

$$
b_{1}<b_{2}<b_{3}<\ldots
$$

We let $U$ denote the set of elements $g \in G_{0}$ such that $\mathrm{v}_{g}\left(b_{2}\right)=+\infty$. We can assume that $U$ is the largest possible. For every $a^{\prime} \in Y, a^{\prime} \leq \sup _{n}\left(b_{n}\right)$, and every $g \in G_{0}$, we can find some $n(g) \in \mathbf{N}$ such that $\mathbf{v}_{g}\left(a^{\prime}\right) \leq \mathbf{v}_{g}\left(b_{n(g)}\right)$, so $a^{\prime} \leq b_{n}$, where $n=\max _{g \in G_{0}} n(g)$, and $a^{\prime} \in \mathcal{A}$. Therefore $\sup _{n}\left(b_{n}\right) \in \mathcal{B}$. By the maximality of $U$ we have $\mathrm{v}_{g}\left(\sup _{n}\left(b_{n}\right)\right)<+\infty$ for all $g \in G_{0} \backslash U$, contradicting (10). We have shown (9) and the assertion follows, as $\mathcal{B}_{\max }$ is finite by Lemma 5 .

(ii) $\Rightarrow$ ( $i$ ii $),(8)$ etc. We assume the $B_{i}$ s non-empty and we can have $\mathrm{v}_{g}\left(\alpha_{i, 1}\right)=$ $\mathrm{v}_{g}\left(\alpha_{i, 2}\right)$ for each $i \in\{1, \ldots, m\}$ and $g \in G_{0}$ such that $\mathrm{v}_{g}\left(\alpha_{i, 2}\right)<+\infty$, by splitting each $B_{i}$ in (ii) to finitely many summands if necessary. For each $B_{i}$ we put

$$
V_{i}=\left\{g \in G_{0}: v_{g}\left(\alpha_{i, 2}\right)=+\infty\right\}
$$

and

$$
\beta_{i}=\prod_{g \in G_{0} \backslash V_{i}} g^{\mathrm{v}_{g}\left(\alpha_{i, 2}\right)} \in \mathcal{F}\left(G_{0} \backslash V_{i}\right) .
$$

We have $\left(V_{i}, \beta_{i}, y_{i}\right) \in \mathrm{S}\left(G_{0}\right)$ and

$$
B_{i}=\Omega_{y_{i}}\left(V_{i}, \beta_{i}\right) \backslash R_{i}
$$

for some set $R_{i}$ equal to a finite union of proper sub-cubes of $\Omega_{y_{i}}\left(V_{i}, \beta_{i}\right)$. We can group the $B_{i}$ s with equal triples $\left(V_{i}, \beta_{i}, y_{i}\right)$, and possibly re-order them, so that for some $m^{\prime} \leq m$ we have 


$$
\begin{aligned}
\mathcal{A} & =\bigcup_{i=1}^{m^{\prime}} B_{i}^{\prime}, \\
B_{i}^{\prime} & =\Omega_{y_{i}}\left(V_{i}, \beta_{i}\right) \backslash R_{i}^{\prime}, \quad i=1, \ldots, m^{\prime},
\end{aligned}
$$

where $\left(V_{i}, \beta_{i}, y_{i}\right) \neq\left(V_{j}, \beta_{j}, y_{j}\right)$ for all $i, j \in\left\{1, \ldots, m^{\prime}\right\}, i \neq j$, and each set $R_{i}^{\prime}$ is equal to a finite union of sub-cubes of $\Omega_{y_{i}}\left(V_{i}, \beta_{i}\right)$ of lower rank. By the inclusionexclusion principle we obtain

$$
\mathcal{A}=\sum_{i=1}^{m^{\prime}}\left(\Omega_{y_{i}}\left(V_{i}, \beta_{i}\right)-R_{i}^{\prime}\right)+\sum_{\substack{I \subseteq\left\{1, \ldots, m^{\prime}\right\} \\|I| \geq 2}}(-1)^{1+|I|} \bigcap_{i \in I} B_{i}^{\prime}
$$

and further

$$
\mathcal{A}=\sum_{i=1}^{m^{\prime}} \Omega_{y_{i}}\left(V_{i}, \beta_{i}\right)+R,
$$

where $R$ is a combination of characteristic functions of proper sub-cubes of the $\Omega_{y_{i}}\left(V_{i}, \beta_{i}\right)$. We obtain (iii). Uniqueness of (7) follows from Lemma 2.4. By Lemmas 3 and 4 we have $\mathrm{rk} B_{i}^{\prime}=\left|V_{i}\right|$ and $\operatorname{deg} B_{i}^{\prime}=\left|\beta_{i}\right|, i=1, \ldots, m^{\prime}$, so (8) follows. Finally we note that every summand of $R_{i}^{\prime}$ in (11) is a proper subset one of the cubes $\Omega_{y_{i}}\left(V_{i}, \beta_{i}\right)$, and so is every intersection $\bigcap_{i \in I} B_{i}^{\prime}$ in (11). Hence the cubes $\Omega_{y_{i}}\left(V_{i}, \beta_{i}\right)$ are the only inclusion-maximal sets in (12), and they appear with the coefficient 1 there.

(iii) $\Rightarrow$ (i). Suppose $l \geq n+1$ and $a_{1}, a_{3}, \ldots, a_{2 l-1} \in \mathcal{A}, a_{2}, a_{4}, \ldots, a_{2 l-2} \in$ $\mathcal{F}\left(G_{0}\right) \backslash \mathcal{A}$ are such that $a_{1}\left|a_{2}\right| a_{3}|\ldots| a_{2 l-1}$ and for some $y \in G$ we have $\sigma\left(a_{i}\right)=y, i=1, \ldots, 2 l-1$. For $i=1, \ldots, 2 l-1$ and $j=1, \ldots, n$, let $c_{i j}$ denote the value of the characteristic function of $\Omega_{g_{j}}\left(U_{j}, S_{j}\right)$ on $a_{i}$, and let $c_{2 l, j}=0$. No two adjacent rows of the matrix $\left[c_{i j}\right]_{\substack{i=1, \ldots, 2 l \\ j=1, \ldots, n}}$, are equal as, by (7), it would place two subsequent $a_{i}$ s both in $\mathcal{A}$ or outside of $\mathcal{A}$, or it would imply $a_{2 l-1} \notin \mathcal{A}$. By the Dirichlet's box principle there exists some $j \in\{1, \ldots, n\}$ such that $c_{i j} \neq c_{i+1, j}$ for at least three different values of $i$. However this is impossible by $l\left(\Omega_{g_{j}}\left(U_{j}, S_{j}\right)\right)=1$. Hence $l(\mathcal{A}) \leq n$.

Given (7) and $r \in \mathbf{N}_{0}$ we call the number

$$
\operatorname{deg}_{r}(\mathcal{A})= \begin{cases}\max _{j:\left|U_{j}\right| \geq r}\left|S_{j}\right|, & 0 \leq r \leq \mathrm{rk} \mathcal{A} \\ 0, & r>\operatorname{rk} \mathcal{A}\end{cases}
$$

the rank- $r$ degree of $\mathcal{A}$. The rank- 0 degree will also be called the absolute degree of $\mathcal{A}$. We have $\operatorname{deg}(\mathcal{A})=\operatorname{deg}_{\mathrm{rk} \mathcal{A}}(\mathcal{A}) \leq \operatorname{deg}_{\mathrm{rk} \mathcal{A}-1}(\mathcal{A}) \leq \ldots \leq \operatorname{deg}_{1}(\mathcal{A}) \leq \operatorname{deg}_{0}(\mathcal{A})$ for $\mathcal{A} \neq \emptyset$, and $\operatorname{deg}_{0}(\emptyset)=0$. We also put $\operatorname{deg}_{-\infty}(\mathcal{A})=+\infty$ for all $\mathcal{A} \subseteq \mathcal{F}\left(G_{0}\right)$ with $l(\mathcal{A})<+\infty$, so that the equality $\operatorname{deg}(\mathcal{A})=\operatorname{deg}_{\mathrm{rk}} \mathcal{A}(\mathcal{A})$ holds for $\mathcal{A}=\emptyset$ as well. We say that an $\Omega$ set $\mathcal{A}$ is of absolute degree 0 if $l(\mathcal{A})<+\infty$ and $\operatorname{deg}_{0}(\mathcal{A})=0$. 


\subsection{Singular sets}

If $\mathcal{A} \subseteq \mathcal{F}\left(G_{0}\right), l(\mathcal{A})<+\infty, r \in \mathbf{N}_{0}$ and $d \in \mathbf{N}$, then we say that $\mathcal{A}$ is $(r, d)$-singular if the unique representation (7) satisfies the following conditions:

(i) There is at least one $j \in\{1, \ldots, n\}$ such that

$$
\left|U_{j}\right|=r \text { and }\left|S_{j}\right|=d
$$

(ii) The sign of $\gamma_{j}$ is the same for every $j$ satisfying (13).

(iii) For each $j \in\{1, \ldots, n\}$ not satisfying (13) we have $\left|U_{j}\right|<r$ or $\left|S_{j}\right|<d$.

We note that the condition $(i i)$ is non-trivial: although the $(r, d)$ pair is maximal among the pairs $\left(\operatorname{rk} \Omega_{g_{j}}\left(U_{j}, S_{j}\right), \operatorname{deg} \Omega_{g_{j}}\left(U_{j}, S_{j}\right)\right.$ ) in the product order by $(i)$ and (iii) alone, it need not be maximal in the lexicographic order, hence the summands in (7) satisfying (13) need not be inclusion-maximal. The interplay between these two orders is really the root of the problem of showing that a set is $(r, d)$-singular for some suitable $r$ and $d$. In the remainder of this subsection we solve this problem in the cases $\operatorname{deg} \mathcal{A}>0($ Fact 7$)$ and $\mathcal{A}=\operatorname{Div}(\mathcal{A})$ (Theorem 11).

Fact 7 If $\mathcal{A} \subseteq \mathcal{F}\left(G_{0}\right)$ is such that $\mathcal{A} \neq \emptyset, l(\mathcal{A})<\infty$ and $\operatorname{deg} \mathcal{A}>0$, then $\mathcal{A}$ is $(\operatorname{rk} \mathcal{A}, \operatorname{deg} \mathcal{A})$-singular.

Proof We can take $r=\operatorname{rk} \mathcal{A}$ and $d=\operatorname{deg} \mathcal{A}$. Conditions (i) and (iii) hold by Proposition 6. By (8) and Lemma 2.2 any set $\Omega_{g_{j}}\left(U_{j}, S_{j}\right)$ in (7) satisfying $\left|U_{j}\right|=r$ is inclusion-maximal, so $\gamma_{j}=1$, hence condition ( $i i$ ) holds as well.

We define an equivalence relation in $\mathcal{F}\left(G_{0}\right)$ by

$$
S \sim S^{\prime} \Leftrightarrow \sigma(S)=\sigma\left(S^{\prime}\right) \wedge \operatorname{Supp}(S)=\operatorname{Supp}\left(S^{\prime}\right)
$$

for $S, S^{\prime} \in \mathcal{F}\left(G_{0}\right)$. We denote by $[S]_{\sim}$ the equivalence class of $S$. For $\mathcal{A} \subseteq \mathcal{F}\left(G_{0}\right)$ we put

$$
\operatorname{Ground}(\mathcal{A})=\{S \in \mathcal{A}:[S] \sim \subseteq \mathcal{A}\}
$$

and

$$
\operatorname{Elev}(\mathcal{A})=\mathcal{A} \backslash \operatorname{Ground}(\mathcal{A})
$$

Lemma 8 A set $\mathcal{A} \subseteq \mathcal{F}\left(G_{0}\right)$ is of absolute degree 0 if and only if it is a union of equivalence classes of the relation $\sim$.

Proof For $g \in G, U \subseteq G_{0}$ the set

$$
\Omega_{g}(U, 1,1)=\left\{S \in \mathcal{F}\left(G_{0}\right): \sigma(S)=g, \operatorname{Supp}(S)=U\right\}
$$


is either empty or is an equivalence class of $\sim$. By substituting

$$
\Omega_{g_{j}}\left(U_{j}, 1\right)=\bigcup_{V \subseteq U_{j}} \Omega_{g_{j}}(V, 1,1)
$$

in (7) we get the implication $(\Rightarrow)$. To get the converse, we first note that for $S \in \mathcal{F}\left(G_{0}\right)$, $g=\sigma(S)$ and $U=\operatorname{Supp}(S)$ we have

$$
[S]_{\sim}=\Omega_{g}(U, 1,1)=\Omega_{g}(U, 1) \backslash \bigcup_{V \subsetneq U} \Omega_{g}(V, 1)
$$

so by the inclusion-exclusion principle and Lemma 1.1 we have

$$
\operatorname{deg}_{0}\left([S]_{\sim}\right)=0
$$

If $\mathcal{A}$ is a union of equivalence classes of $\sim$, then it is a finite union, as there are only finitely many such classes in $\mathcal{F}\left(G_{0}\right)$. Therefore

$$
\mathcal{A}=\sum_{i=1}^{m}\left[S_{i}\right]_{\sim}
$$

for some $m \in \mathbf{N}_{0}$ and $S_{1}, \ldots, S_{m} \in \mathcal{F}\left(G_{0}\right)$, where addition is in terms of characteristic functions, hence the unique representations may likewise be added, and $\operatorname{deg}_{0}(\mathcal{A})=0$ follows from (14).

Corollary 9 If $\mathcal{A} \subseteq \mathcal{F}\left(G_{0}\right)$, then the set $\operatorname{Ground}(\mathcal{A})$ is the largest $\Omega$ set of absolute degree 0 contained in $\mathcal{A}$. In particular, we have $\operatorname{Elev}(\mathcal{A})=\emptyset$ if and only if $\operatorname{deg}_{0}(\mathcal{A})=0$.

Lemma 10 Let $\mathcal{A}$ be an $\Omega$ set satisfying $\mathcal{A}=\operatorname{Div}(\mathcal{A})$. Then

$$
\operatorname{deg}(\operatorname{Elev}(\mathcal{A}))>0
$$

Proof We have $l(\mathcal{A}) \leq 1$. If $\operatorname{deg}_{0}(\mathcal{A})=0$, then $\operatorname{Elev}(\mathcal{A})=\varnothing$ by Corollary 9 , so $\operatorname{deg}(\operatorname{Elev}(\mathcal{A}))=+\infty$. Suppose $\operatorname{deg}_{0}(\mathcal{A})>0$ and let

$$
\operatorname{Elev}(\mathcal{A})=\sum_{j=1}^{n} \gamma_{j} \cdot \Omega_{g_{j}}\left(U_{j}, S_{j}\right)
$$

with $n \geq 1$, be the unique representation of $\operatorname{Elev}(\mathcal{A})$. Suppose as we may (by Proposition 6 and possible re-ordering of (15)) that $\gamma_{1} \cdot \Omega_{g_{1}}\left(U_{1}, S_{1}\right)$ is one of the summands with

$$
\left|U_{1}\right|=\operatorname{rk}(\operatorname{Elev}(\mathcal{A})) \quad \text { and } \quad\left|S_{1}\right|=\operatorname{deg}(\operatorname{Elev}(\mathcal{A})) .
$$


It suffices to show that $S_{1} \neq 1$, so we assume the converse. The set $\Omega_{g_{1}}\left(U_{1}, 1\right)$ is inclusion-maximal among the summands of (15), again by Proposition 6 and Lemma 2.2, hence $\gamma_{1}=1$. Moreover this maximality implies, by Lemma 2.3, that for every $d \in \mathbf{N}_{0}$ there exists some $S \in \Omega_{g_{1}}\left(U_{1}, 1, d\right)$ such that

$$
S \notin \bigcup_{j=2}^{n} \Omega_{g_{j}}\left(U_{j}, S_{j}\right)
$$

and thus $S \in \operatorname{Elev}(\mathcal{A})$ by (15). It follows from Lemma 2.1 that

$$
\Omega_{g_{1}}\left(U_{1}, 1\right) \subseteq \operatorname{Div}(\operatorname{Elev}(\mathcal{A})) \subseteq \operatorname{Div}(\mathcal{A})=\mathcal{A}
$$

Then $\Omega_{g_{1}}\left(U_{1}, 1\right) \subseteq \operatorname{Ground}(\mathcal{A})$ by Corollary 9 , so $\Omega_{g_{1}}\left(U_{1}, 1\right) \cap \operatorname{Elev}(\mathcal{A})=\emptyset$, contradicting $S \in \Omega_{g_{1}}\left(U_{1}, 1\right) \cap \operatorname{Elev}(\mathcal{A})$.

Theorem 11 Let $\mathcal{A}$ be an $\Omega$ set such that $\operatorname{Div}(\mathcal{A})=\mathcal{A}$. Then the following conditions are equivalent:

(i) $\mathcal{A}$ is $(r, d)$-singular for some $r \in \mathbf{N}_{0}$ and $d \in \mathbf{N}$,

(ii) $\operatorname{deg}_{0}(\mathcal{A})>0$.

Proof $(i) \Rightarrow$ (ii) By definition, we have $\operatorname{deg}_{r}(\mathcal{A})=d$, so $\operatorname{deg}_{0}(\mathcal{A}) \geq d>0$.

(ii $) \Rightarrow($ i $)$ Since $\operatorname{deg}_{0}(\mathcal{A})>0$, we have $\operatorname{Elev}(\mathcal{A}) \neq \emptyset$ by Corollary 9 . We also have $\operatorname{deg}(\operatorname{Elev}(\mathcal{A}))>0$ by $\operatorname{Lemma} 10$, so the $\operatorname{set} \operatorname{Elev}(\mathcal{A})$ is $(r, d)$-singular for $r=$ $\operatorname{rk}(\operatorname{Elev}(\mathcal{A}))$ and $d=\operatorname{deg}(\operatorname{Elev}(\mathcal{A}))$ by Fact 7 . We have

$$
\mathcal{A}=\operatorname{Ground}(\mathcal{A})+\operatorname{Elev}(\mathcal{A})
$$

where addition is in terms of characteristic functions, hence the unique representation (7) of $\mathcal{A}$ may be obtained by adding the unique representations of $\operatorname{Ground}(\mathcal{A})$ and $\operatorname{Elev}(\mathcal{A})$. We have $\operatorname{deg}_{0}(\operatorname{Ground}(\mathcal{A}))=0$ by Corollary 9 , so $\mathcal{A}$ is $(r, d)$-singular as well.

\section{$4 \Omega$ sets in semigroups with divisor theory}

Let $H$ be a commutative, cancellative semigroup with a unit, with divisor theory $\varphi: H \rightarrow \mathcal{F}(P)$ and a finite class group $G$. For $a \in \mathcal{F}(P)$ the divisor class of $a$ is denoted as $[a]$. We use multiplicative notation for $H$. Let $h=|G|$ and let $G_{0} \subseteq G$ be the set of classes that contain at least one prime divisor. As usual, $\Omega_{g}(a)$ denotes (for $\left.a \in \mathcal{F}(P), g \in G_{0}\right)$ the number of prime divisors of $a$ in the class $g$ counted according to their multiplicities, cf. [4, Example 9.2.7] and, for the origins of this notation, [1,6]. Let $\tilde{\beta}: \mathcal{F}(P) \rightarrow \mathcal{F}\left(G_{0}\right)$,

$$
\tilde{\beta}(a)=\prod_{g \in G_{0}} g^{\Omega_{g}(a)} .
$$


A set $A \subseteq \mathcal{F}(P)$ is an $\Omega$ set if and only if $A=\tilde{\beta}^{-1}(\mathcal{A})$ for some $\mathcal{A} \subseteq \mathcal{F}\left(G_{0}\right)$. In that case we define the rank, degree and the number of layers of $A$ as $\operatorname{rk} \mathcal{A}, \operatorname{deg} \mathcal{A}$ and $l(\mathcal{A})$ respectively, and we say that $A$ is $(r, d)$-singular if $\mathcal{A}$ is. We extend this notation and terminology also to the set $\varphi^{-1}(A)$ in case $A \subseteq \varphi(H)$.

\subsection{Elements with at most a given number of factorization lengths}

We are going to show that the set $\boldsymbol{G}_{k}$ (for a positive integer $k$ ) is $(r, d)$-singular for some suitable $r$ and $d$ unless $\boldsymbol{G}_{k}=\boldsymbol{G}_{1}=H$. First we recall some well-known facts related to $\boldsymbol{G}_{k}$. The set $U$ is called half-factorial if the monoid $\mathcal{B}(U)=\Omega_{0}(U, 1)$ is half-factorial, i.e. every element of $\mathcal{B}(U)$ has a unique length of factorization into irreducibles. The homomorphism

$$
\beta: H \rightarrow \mathcal{B}\left(G_{0}\right), \quad \beta(a)=\tilde{\beta}(\varphi(a)),
$$

is a transfer homomorphism [4, Definition 3.2.1], so it establishes a one-to-one correspondence between lengths of factorizations of $a$ in $H$ and $\beta(a)$ in $\mathcal{B}\left(G_{0}\right)$, hence $\boldsymbol{G}_{k}=\beta^{-1}\left(\beta\left(\boldsymbol{G}_{k}\right)\right)$ is an $\Omega$ set. We have $\operatorname{Div}\left(\boldsymbol{G}_{k}\right)=\boldsymbol{G}_{k}$, in particular $l\left(\boldsymbol{G}_{k}\right)=1$. The following equivalence has appeared in several works at various levels of generality. We provide a proof for the convenience of the reader.

Lemma 12 (cf., e.g., [22,23], [4, Proposition 6.7.3.1], [4, Theorem 9.4.6, Assertion A1]). Let $U \subseteq G_{0}$. The following conditions are equivalent:

(i) $U$ is half-factorial,

(ii) $\Omega_{0}(U, 1) \subseteq \beta\left(\boldsymbol{G}_{1}\right)$,

(iii) $\Omega_{0}(U, S) \subseteq \beta\left(\boldsymbol{G}_{k}\right)$ for some $k \in \mathbf{N}$ and $S \in \mathcal{F}\left(G_{0}\right)$ such that $(U, S, 0) \in$ $\mathrm{S}\left(G_{0}\right)$,

(iv) for every irreducible $a \in \beta^{-1}\left(\Omega_{0}(U, 1)\right)$ we have

$$
\mathrm{k}(a):=\sum_{g \in G_{0}} \frac{\Omega_{X}(a)}{\operatorname{ord} g}=1 .
$$

Proof $(i) \Rightarrow$ (ii). If $a \in \beta^{-1}\left(\Omega_{0}(U, 1)\right)$, then $\beta(a) \in \mathcal{B}(U)$ has a unique factorization length in $\mathcal{B}\left(G_{0}\right)$, so $a \in \boldsymbol{G}_{1}$.

(ii) $\Rightarrow$ (iii) is clear.

(iii) $\Rightarrow$ (iv). We have $\operatorname{Div}\left(\Omega_{0}(U, S)\right) \subseteq \operatorname{Div}\left(\beta\left(\boldsymbol{G}_{k}\right)\right)=\beta\left(\boldsymbol{G}_{k}\right)$ and $\Omega_{0}(U, 1) \subseteq$ $\operatorname{Div}\left(\Omega_{0}(U, S)\right)$ by Lemma 2.1 , so $\Omega_{0}(U, 1) \subseteq \beta\left(\boldsymbol{G}_{k}\right)$. Suppose $a \in \beta^{-1}\left(\Omega_{0}(U, 1)\right)$ is irreducible and $\mathrm{k}(a) \neq 1$. Let $\varphi(a)=\prod_{i=1}^{n} p_{i}^{\alpha_{i}}$ be the factorization of $\varphi(a)$ to prime divisors, and let $a_{1}, \ldots, a_{n} \in H$ be some irreducibles satisfying $\varphi\left(a_{i}\right)=p_{i}^{\text {ord }\left[p_{i}\right]}$. Then the associated elements $a^{h} \sim \prod_{i=1}^{n} a_{i}^{h \alpha_{i} / \operatorname{ord}\left[p_{i}\right]}$ have factorizations of lengths $h$ and $h \mathrm{k}(a)$, so $a^{k h}$ has at least $k+1$ factorizations of distinct lengths. Hence $a \notin \boldsymbol{G}_{k}$, a contradiction.

(iv) $\Rightarrow(i)$. Every element of $\mathcal{B}(U)$ is of the form $\beta(a)$ for some $a \in H$, $\operatorname{Supp}(\beta(a)) \subseteq U$. If $a=a_{1} \ldots a_{n}$ is a factorization of such $a$ to irreducibles, then $n=\sum_{i=1}^{n} \mathrm{k}\left(a_{i}\right)=\mathrm{k}(a)$, so $a$ and $\beta(a)$ have unique factorization length. 
Theorem 13 Let $k$ be a positive integer. The set $\boldsymbol{G}_{k}$ is $(r, d)$-singular for some $r \in \mathbf{N}_{0}$ and $d \in \mathbf{N}$ if and only if the set $G_{0}$ is not half-factorial.

Proof If $G_{0}$ is half-factorial, then by Lemma 12 we have $\beta(H)=\Omega_{0}\left(G_{0}, 1\right) \subseteq \boldsymbol{G}_{1}$, so $\boldsymbol{G}_{k}=\boldsymbol{G}_{1}=H$. Suppose $G_{0}$ is not half-factorial. Then we can find an irreducible $a \in H$ such that $\mathrm{k}(a) \neq 1$. Of course $a \in \boldsymbol{G}_{1} \subseteq \boldsymbol{G}_{k}$. If

$$
[\beta(a)]_{\sim}=\Omega_{0}(\operatorname{Supp}(\beta(a)), 1,1) \subseteq \beta\left(\boldsymbol{G}_{k}\right)
$$

then

$$
\Omega_{0}(\operatorname{Supp}(\beta(a)), 1)=\operatorname{Div}\left(\Omega_{0}(\operatorname{Supp}(\beta(a)), 1,1)\right) \subseteq \beta\left(\boldsymbol{G}_{k}\right)
$$

so $\operatorname{Supp}(\beta(a))$ is half-factorial by Lemma 12 , a contradiction. Hence

$$
\Omega_{0}(\operatorname{Supp}(\beta(a)), 1,1) \nsubseteq \beta\left(\boldsymbol{G}_{k}\right)
$$

and $\operatorname{deg}_{0}\left(\boldsymbol{G}_{k}\right)>0$ by Lemma 8 . The assertion follows from Theorem 11 .

4.2 Elements without divisors in a given $\Omega$ set

Let $F \subseteq \mathcal{F}(\mathcal{P})$ denote a non-empty $\Omega$ set of "forbidden" divisors. The set

$$
A=\mathcal{F}(\mathcal{P}) \backslash F \mathcal{F}(\mathcal{P})=\{a \in \mathcal{F}(\mathcal{P}): b \nmid a \text { for all } b \in F\}
$$

is an $\Omega$ set and it includes all divisors of its elements. The computation of $\operatorname{rk} A$ and $\operatorname{deg} A$ may be non-trivial, but we can usually determine whether $\operatorname{deg}_{0}(A)$ is positive or not. Let $F_{\min }$ denote the set of minimal elements (with respect to division) of $F$.

Theorem 14 If $F \subseteq \mathcal{F}(\mathcal{P})$ is an $\Omega$ set, then the set $A=\mathcal{F}(\mathcal{P}) \backslash F \mathcal{F}(\mathcal{P})$ is $(r, d)$ singular for some $r \in \mathbf{N}_{0}$ and $d \in \mathbf{N}$ if and only if

$$
\max _{b \in F_{\min }} \max _{g \in G_{0}} \Omega_{g}(b)>1 \text {. }
$$

Proof The set $F_{\min }$ is an $\Omega$ set, as $F$ is. We have $F \mathcal{F}(\mathcal{P})=F_{\min } \mathcal{F}(\mathcal{P})$, because divisibility is a well-order on $\mathcal{F}(\mathcal{P})$. The fact that $F_{\min }$ is an antichain implies that $l\left(F_{\min }\right)=1$ and, using Lemma 5 , also

$$
\tilde{\beta}\left(F_{\min }\right)=\bigcup_{j=1}^{n}\left\{S_{j}\right\}
$$

for some $S_{j} \in \mathcal{F}\left(G_{0}\right), j=1, \ldots, n$. Let $\mathcal{A}=\tilde{\beta}(A)$ and $V_{j}=\operatorname{Supp}\left(S_{j}\right)$. Then

$$
\mathcal{A}=\bigcup_{(U, S, y) \in \mathrm{S}(\mathcal{A})} \Omega_{y}(U, S)
$$


where $\mathrm{S}(\mathcal{A})$ consists of all triples $(U, S, y) \in \mathrm{S}\left(G_{0}\right)$ such that for every $j$ there is at least one $g \in V_{j} \backslash U$ with $\mathrm{v}_{g}(S)<\mathrm{v}_{g}\left(S_{j}\right)$. In that case $U$ does not contain any of the $V_{j}$ and, conversely, for every $U$ that does not contain any of the $V_{j}$ we have $(U, 1,0) \in \mathrm{S}(\mathcal{A})$. By Lemma 8 we have $\operatorname{deg}_{0}(\mathcal{A})=0$ if and only if $\mathcal{A}$ is a union of equivalence classes of the relation $\sim$. Let

$$
m=\max _{1 \leq j \leq n} \max _{g \in G_{0}} \mathrm{v}_{g}\left(S_{j}\right) .
$$

If $m=0$, then $\tilde{\beta}\left(F_{\min }\right)=\{1\}$ and $\mathcal{A}=\emptyset$. If $m=1$, then for every $S \in \mathcal{A}$ we have $V_{j} \nsubseteq \operatorname{Supp}(S), j=1, \ldots, n$, so

$$
[S]_{\sim}=\Omega_{\sigma(S)}(\operatorname{Supp}(S), 1,1) \subseteq \mathcal{A}
$$

Hence $\operatorname{deg}_{0}(\mathcal{A})=0$. Finally, if $m>1$, let us fix $j$ and $g \in G_{0}$ with $\mathrm{v}_{g}\left(S_{j}\right)=m$. Let $S=g^{-1} S_{j} \in \mathcal{F}\left(G_{0}\right)$, so $\operatorname{Supp}(S)=V_{j}$, and let $y=\sigma(S)$. We have $S \in \mathcal{A}$, because $S_{j} \in \tilde{\beta}\left(F_{\min }\right)$. If

$$
[S]_{\sim}=\Omega_{y}\left(V_{j}, 1,1\right) \subseteq \mathcal{A}
$$

then we would have $V_{j} \subseteq U$, a contradiction. Hence $\operatorname{deg}_{0}(\mathcal{A})>0$ and the assertion follows by Theorem 11 .

Acknowledgments The author wishes to thank Professor Jerzy Kaczorowski for his helpful remarks during the preparation of this manuscript. The author also thanks Professor Władysław Narkiewicz for the information on the earliest use of the $\Omega$ notation. The author gratefully acknowledges the remarks of the anonymous referee, that helped improve the presentation of the paper. The referee's suggestions have also helped make the notation compatible with other recent works on the subject. This work has been supported by the Polish National Science Centre Grant number N N201 605940.

Open Access This article is distributed under the terms of the Creative Commons Attribution License which permits any use, distribution, and reproduction in any medium, provided the original author(s) and the source are credited.

\section{References}

1. Delange, H.: Sur la distribution des entiers ayant certaines propriétés. Ann. Sci. Ecole Norm. Sup. 3(73), 15-74 (1956)

2. Fogels, E.: Zur Arithmetik quadratischer Zahlenkörper. Univ. Riga. Wiss. Abh. Kl. Math. Abt. 1, 23-47 (1943)

3. Geroldinger, A., Halter-Koch, F.: Realization theorems for semigroups with divisor theory. Semigroup Forum 44(2), 229-237 (1992)

4. Geroldinger, A., Halter-Koch, F.: Non-unique factorizations. Chapman \& Hall/CRC, Boca Raton (2006)

5. Halter-Koch, F.: Halbgruppen mit Divisorentheorie. Expo. Math. 8(1), 27-66 (1990)

6. Hardy, G.H., Wright, E.M.: An introduction to the theory of numbers. Clarendon Press, Oxford (1938)

7. Kaczorowski, J.: Some remarks on factorization in algebraic number fields. Acta Arith. 43(1), 53-68 (1983)

8. Kaczorowski, J., Perelli, A.: Functional independence of the singularities of a class of Dirichlet series. Am. J. Math. 120(2), 289-303 (1998) 
9. Kaczorowski, J., Pintz, J.: Oscillatory properties of arithmetical functions. II. Acta Math. Hung. 49(34), 441-453 (1987)

10. Narkiewicz, W.: On algebraic number fields with non-unique factorization. Colloq. Math. 12, 59-68 (1964)

11. Narkiewicz, W.: On algebraic number fields with non-unique factorization. II. Colloq. Math. 15, 49-58 (1966)

12. Narkiewicz, W.: Numbers with unique factorization in an algebraic number field. Acta Arith. 21, 313-322 (1972)

13. Narkiewicz, W.: Elementary and analytic theory of algebraic numbers. PWN-Polish Scientific Publishers, Warsaw (1974). Monografie Matematyczne, Tom 57

14. Narkiewicz, W.: Finite abelian groups and factorization problems. Colloq. Math. 42, 319-330 (1979)

15. Narkiewicz, W.: Numbers with all factorizations of the same length in a quadratic number field. Colloq. Math. 45(1):71-74 (1981)

16. Radziejewski, M.: On the distribution of algebraic numbers with prescribed factorization properties. Acta Arith. 116(2), 153-171 (2005)

17. Radziejewski, M.: Oscillations of error terms associated with certain arithmetical functions. Monatsh. Math. 144(2), 113-130 (2005)

18. Radziejewski, M.: Independence of Hecke zeta functions of finite order over normal fields. Trans. Am. Math. Soc. 359(5), 2383-2394 (2007)

19. Radziejewski, M.: Oscillatory properties of real functions with weakly bounded Mellin transform. Q. J. Math. 65(1), 249-266 (2014)

20. Radziejewski, M.: Algebraic independence of logarithmic singularities of some complex functions. J. Math. Anal. Appl. 410(2), 764-770 (2014)

21. Radziejewski, M., Schmid, W.A.: On the asymptotic behavior of some counting functions. Colloq. Math. 102(2), 181-195 (2005)

22. Skula, L.: On $c$-semigroups. Acta Arith. 31(3), 247-257 (1976)

23. Śliwa, J.: Factorizations of distinct lengths in algebraic number fields. Acta Arith. 31(4), 399-417 (1976)

24. Śliwa, J.: Remarks on factorizations in algebraic number fields. Colloq. Math. 46(1), 123-130 (1982) 